\title{
Design and Implementation of Multi-temporal Image Service System on National Platform for Geospatial Information Services in China
}

\author{
Wang Cong ${ }^{1}$, Zhang Hongping ${ }^{1 *}$ \\ ${ }^{1}$ National Geomatics Center of China, Beijing, China-wangcong@ngcc.cn, zhanghongping@ngcc.cn
}

\author{
Commission IV, WG IV/7
}

KEY WORDS: Massive multi-temporal data; Metadata; Tiles data; System architecture; Object-Relation Database; Cloud services; PostgreSQL

\begin{abstract}
:
With the development of National Platform for Geospatial Information Services in China, it accumulated massive image data with multi-source, multi-temporal and multi-scale. Displaying and taking full use of these historical data can be meaningful to the users. This paper provided design and implementation of multi-temporal image service system based on national platform, which meets the requirements of different users. In this paper, the design of the overall system architecture was introduced, and differe nt system levels stated respectively. The Key technologies presented in the third part, and the implementation displayed parts of functions of this system. Applications, that is typical, recommended in the following part. Also, future efforts and discussions were put forward in the end, which could be guided following work. The research also looks forward to the development of national platform, and the direction of services that should be provided by multi-temporal image service is already under development.
\end{abstract}

\section{INTRODUCTION}

In the information age, web-based geographic information service plays a significant role in the field of public service. National Platform for Geospatial Information Services in China "TIANDITU" has won a place in the public service, especially in the aspects of sharing and distributing information resources around the country. Therefore, the national platform has accumulated massive and large scale data since 2001, when the platform has established.

After years of development, National Platform launched a pilot project for joint construction of national, provincial, and municipal nodes in 2013. As one of the most important projects in National Platform for Geospatial Information Services, data fusion stands at the heart of data update and Platform development. The project aims to integrate the advantages of different data resources such as commercial map data and basic surveying map data to provide freshness and precision service for users.

In the aspect of image service, national platform's traditional image service provides images of 1-18 levels (scale from $1: 295829355$ to $1: 2256$ ). In the Chinese region, the 10-16 level imagery is updated once a year, and the foreign regions are updated according to the satellite data acquisition situation and user needs. The 10-16 level images use the same data source, and the 17-18 level images use the same data source. Since the release of the first version of national node, the image service has been continuously updated, but the old image data has never been fully utilized.

So far, national platform has been accumulated amount of multi-source, multi-temporal and multi-scale image data. So taking full advantages of the historical image data has positive effects on image service, which not only meet demands of users

*Corresponding author from public institutions, government agencies, but also enrich image data service.

\section{RELATED WORKS}

At present, many map services at home and abroad provide multi-temporal image map services. Whether it is a commercial map or a government-supported map, a multitemporal image service provides richer information and a higher user experience for all types of users than a single image service.

Among the global multi-temporal image services, commercial maps represented by Google and Bing provide very highquality multi-temporal image services. Their image service data content is rich, multi-phase time information span is wide, most of the time can be accurate to the day. Its service has a large number of users.

Compared with foreign multi-temporal image maps, China's multi-temporal image services are still relatively backward and single. This is due to the social environment of China's informatization, but more important is the issue of geographic information interoperability and resource sharing. Inconsistent data resource management, inconsistent data standards, and difficulty in sharing data resources have caused difficulties in multi-temporal image map services.

Currently, most of the multi-temporal image services available in China are supported by the government. Among them, the multi-temporal image service of national platform's provincial nodes has emerged and won praise from many users in the industry and abroad.

TIANDITU Fujian, as a sub-node of national platform, provides a very rich multi-temporal image service. The data includes 10-18 levels, the oldest time phase is 2012, and the latest can reach March 2021. It is updated every quarter. Figure 1 shows its multi-temporal image service (Figure 1). 


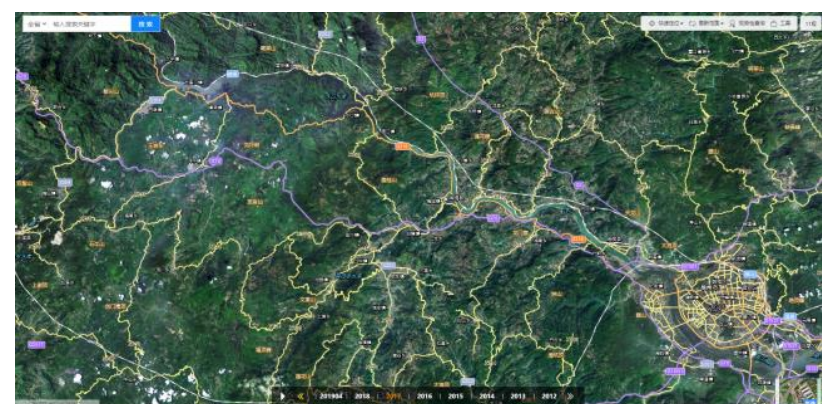

Figure1. Multi-temporal image service of Fujian node

Also as a sub-node of TIANDITU, Shandong's multi-temporal image service provides richer data content and more functions. The image data information can be accurate to the day, and the satellite or sensor information of the query image can be displayed in real time (Figure2).

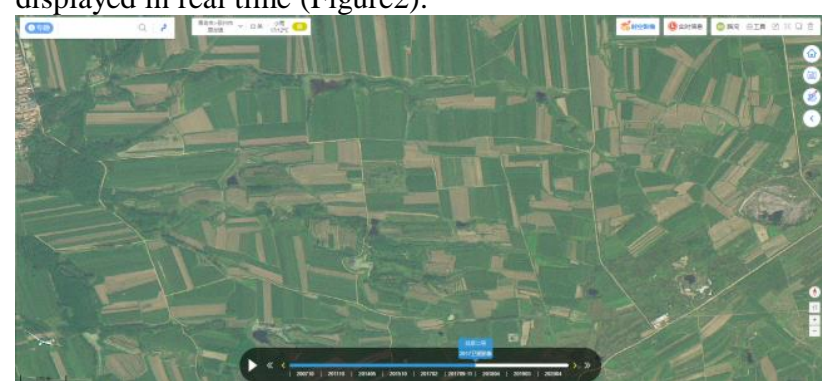

Figure2. Multi-temporal image service of Shandong node

In addition, many government departments and projects have also built multi-temporal image services according to their own business needs. Although it is quite different from the Internet business, its system design and data management methods are still worth learning.

Regardless of whether it is the multi-temporal image service of TIANDITU sub-nodes or the multi-temporal image service constructed by government departments according to business needs, the amount of data is always controllable, and its users also have certain limitations. As a national-level node, it faces multiple domestic and foreign users with multiple data increases. While drawing on other multi-temporal services, we must also have our own innovation and thinking. Only by doing so, can we meet the needs of our own multi-temporal image service.

\section{SYSTEM ARCHITECTURE}

The multi-temporal image service system architecture design (Figure 4) shows that it is comprised of four system levels, which are Cloud services level, data level, system level and user system level. A series of technical standards guide that the overall service system runs with uniform standards and specifications.

\subsection{Cloud Services Level}

The Cloud Services level guarantees that the whole system can run well and safely, moreover, the use of cloud services are in consonance with overall architecture of National Platform for Geospatial Information Services (Figure 3). The public version in running on the public cloud services, and our platform is a Hybrid cloud architecture. The data center of NGCC is directly connected to the cloud data center. Since the size of spatial data are often too big, a subscriber line is used for data update and software testing.

In this study, the cloud services level contains operation system, servers, storages, networks, safe equipment and etc. Compare with traditional system services, cloud services possess high performance in terms of dramatic, stable, efficient, secure data use.

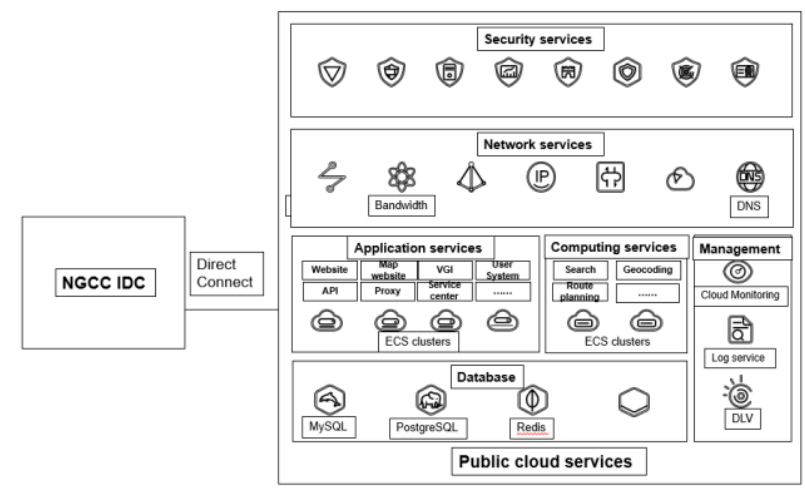

Figure3. Overall architecture of National Platform for Geospatial Information Services

Dramatically, the technology of cloud services could support efficient collaboration services among hundreds of distributed nodes (provincial and municipal nodes) around the country. On the other hand, it could response with high efficiency, too.

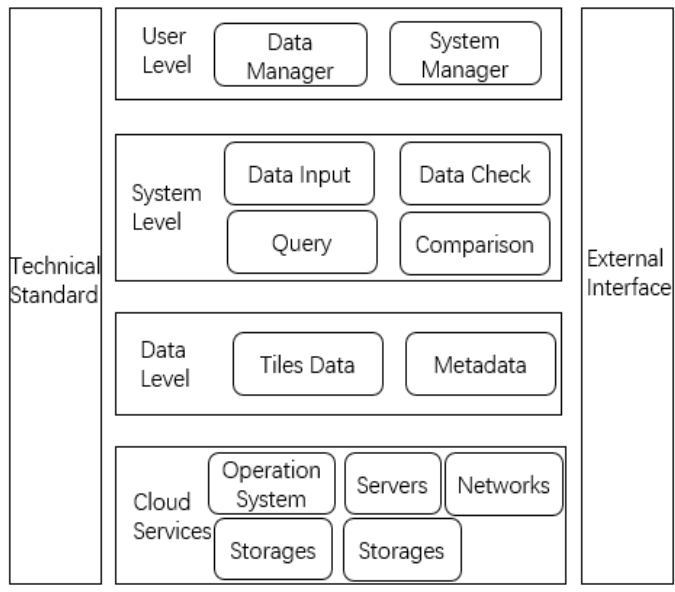

Figure 4. Multi-temporal image service system architecture

\subsection{Data Level}

With the consideration of national platform's technological architecture and image data characteristics, multi-temporal image service employed tiles data to process implement. Besides, the historical remote sensing data, such as aerial images and satellite images, however, plays the role mostly in verification and backups when needs to recall. Hence, the data level just includes tiles data and metadata. The platform obtains its image data from enterprises, public institutions, government agencies of Natural Resources (such as provincial and municipal natural departments) and volunteers. That would easily lead that different organizations, formats, structures and field of data are collected. In order to put the data from different resources in order and standard, Platform Data Standard should be set and published. Although multitemporal image service system has technical standard, the Platform Data Standard, to some extent, is foundation of the 
whole standard. In order to realize the rapid release and sharing application of national platform's multi-temporal image service, the standard specifies a series of data and servicerelated operational details. The metadata involved in the service, the format requirements for the submission of image tile data, the organization method, and the requirements for filling in relevant information are all covered in the table standard. This standard is more applicable to the production and collection of national platform's multi-temporal image service data.

In details, platform Data Standard mainly uses for normalizing tiles data and metadata in the process of data production. As well as, the standard provides normalized edit method and edit content of metadata, which include the region name, code of administrative division, image acquisition time, level, projection type, data production institutions, transducers and remarks. Moreover, all the field has unified name and type. Each different piece of data has exclusive ID, which avoid duplicate data in the database.

In one way, metadata is the most considerable factor in the data level. It reflects the real information of original historical data, especially the acquisition time.

\subsection{System Level}

The system level can support data input, data check (format), compare multi-temporal map and other functions, which will be developed in the future.

Though this service system does not manage the remote sensing data, the nationwide tiles data and metadata still have large size. Consequently, data check plays an important role in data input. It help inspect wrong metadata, for example, the edit errors and field errors. Also, spatial errors in the metadata corresponding to the tiles data can also be detected.

Query function is also conducted in system level. Information such as administrative division region name, code of administrative division, image acquisition time and etc. can be queried based on this function. Figure5 shows the spatial errors detected by system automatically.

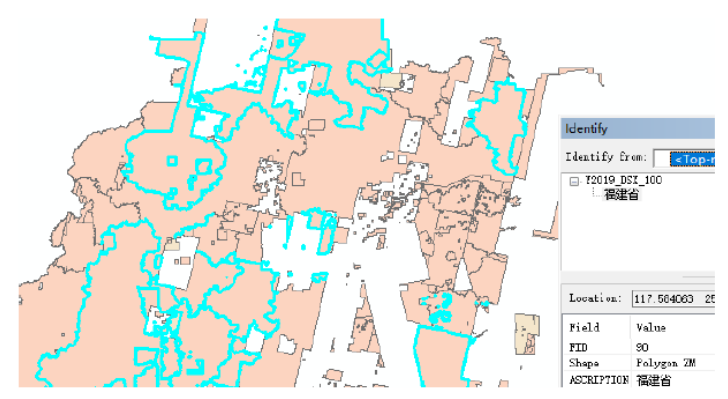

Figure5. Spatial errors detected by system

\subsection{User Level}

Under the user level, data manager and system manager can access their own sub-system by using their own authorities. Separate management for data and system would avoid operation mistakes for different managers. Besides, it is easy to recall operations.

In this level, data management personnel mainly organize multi-temporal data to make it meet the requirements of data storage, and ensure that the metadata information is correct and the metadata corresponds to the real tile data space.

The system manager is responsible for storing the prepared data. When entering the database, the system will automatically check whether each field of the metadata is filled in correctly. If it encounters a filling error, the system will automatically stop writing to the database and notify the administrator of the error. Automatic warehousing inspection is more efficient than manual inspection by data management personnel. If the data is correct, the multi-temporal data will be stored smoothly.

\section{KEY TECHNOLOIES}

\subsection{Efficient Data Management}

Different from general multi-temporal image data management, this system mainly organizes the tiles data and metadata, which improve quick browse and query. PostgreSQL which is the Object-Relation Database, has been used to manage metadata of original image. And the object-based storage managed the tiles data. Accordingly, Elasticsearch has been used in metadata service. Figure 6 shows the key technology of multitemporal image services.

\subsection{Seamless Browsing}

In most image management, remote sensing data would also be stored in the database. For the reason that, it can help to realize the seamless browsing.

This research, however, proposed that corresponding metadata of original remote sensing image to tiles data, which help the metadata information falls on the real spatial place. And the real information of map did not be separated by tiles grid, which realized the seamless browsing.

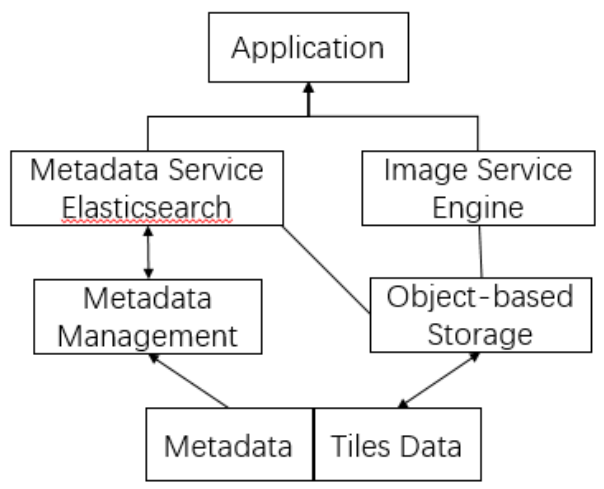

Figure 6. Key technology of service

\section{IMPLEMENTATION}

As mentioned above, the whole implement of system shows that the management of tiles data depends on PostgreSQL, and metadata depends on the Elasticsearch. Moreover, it was developed base on Java. Now the service could support quick browse and query for common users. Furthermore, marking points and polygons could also be used for professional users.

\subsection{Quick Browsing and Query}

This service supports the quick browsing and scan. Users can browse map on the website. And users can browse the map with one screen or comparison mode. Figure 7 shows users can scan map on one screen. Then users can choose different time of image on the website. Figure 8 shows the comparison mode of the multi-temporal image. Users can compare maps of 
different time to find some changes such as houses, settlement places, greenbelts and etc. These changes can be concerned with common and professional users.

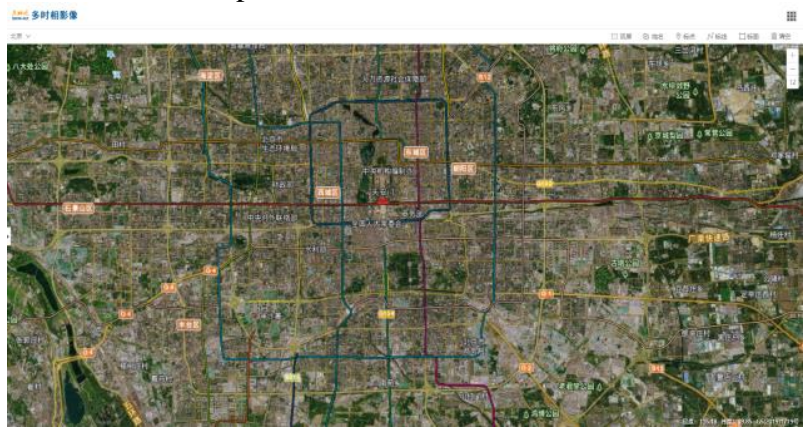

Figure 7. Browse map on one screen

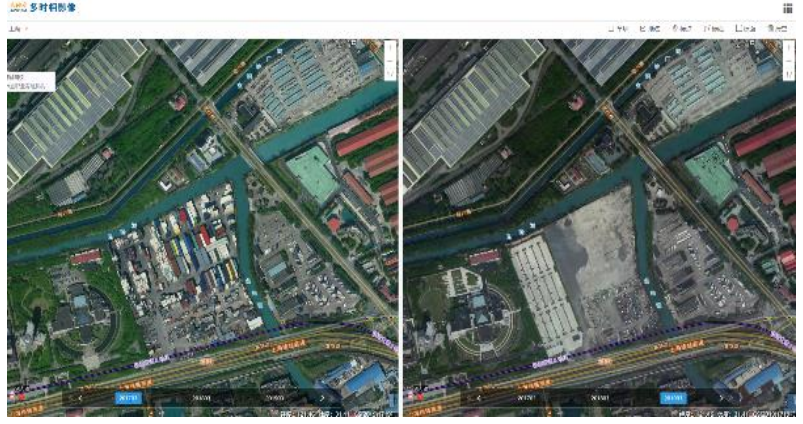

Figure 8. Multi-temporal image Comparison

This system provides quick links of the landmark buildings and some new famous buildings on the left side of website page, which includes Tian'anmen, Shanghai Free Trade Zone, Beijing Daxing International Airport and etc. These links can help users find famous places quickly and understand the development in the certain places. As a result, this implement helps improve experiences for users. Figure 9 shows the landmark buildings list on the left side of website page, and figure 10 shows Shanghai free trade zone in year of 2015 and 2020.

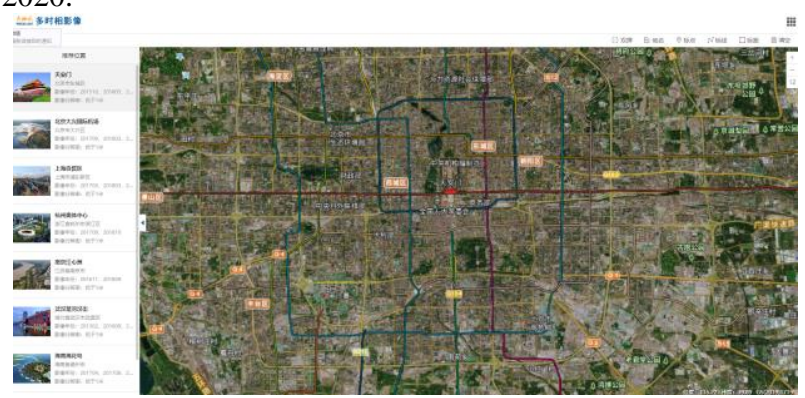

Figure 9. Landmark buildings list

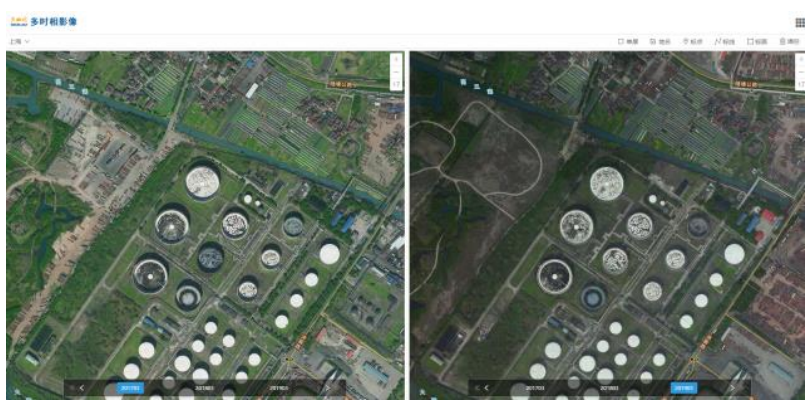

Figure 10. Different time image of Shanghai Free trade zone
In the aspect of query, this system could inquire and search base on administrative places and point of interests (POI), which are in accordance with image service of National Platform for Geospatial Information Services.

\subsection{Data Released}

The first version of multi-temporal image service has released two levels: level 17 and 18, which is the scale of 1:4513 and $1: 2256$. The resolution of remote sensing data is from 0.5 meter to 1 meter. That is the clearest map of public version.

The multi-temporal map only focused on the terrestrial land in china, and area reached 6,300,000 square kilometer. Figure 11 shows the areas that multi-temporal image has covered.

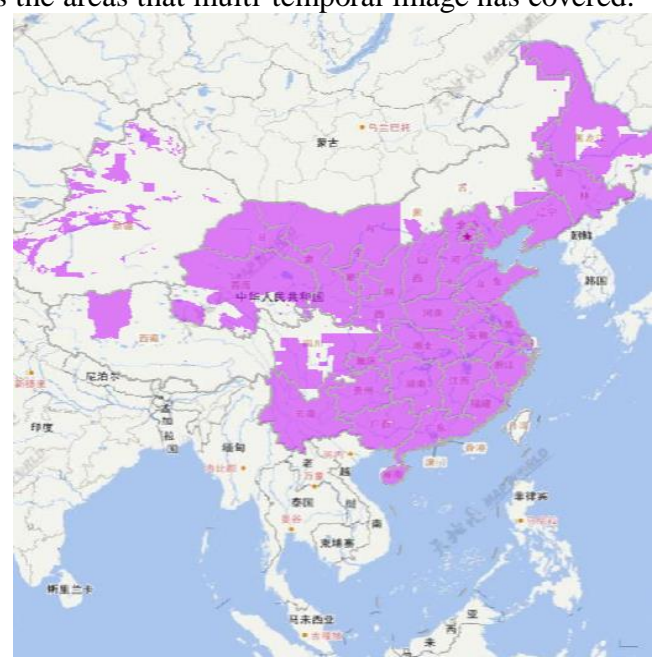

Figure 11 Multi-temporal image has covered

\subsection{Marking Points and Polygons}

The multi-temporal image service system has supported to mark points and polygons. Mostly, this function would be used by professional users. These points and polygons could be exported and used in the professional software. Figure 12 shows that marking points and polygons on the multi-temporal map.

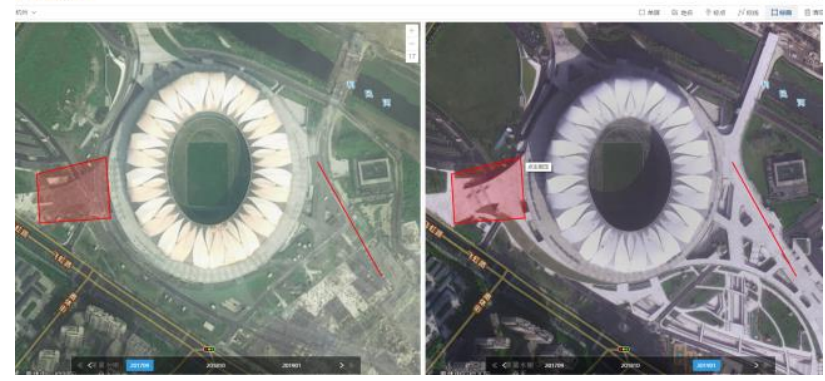

Figure 12. Marking points and polygons

\section{APPLICATIONS}

National Platform for Geospatial Information Services has launched in January 2011. From the perspective of the overall application of national platform, it supports the decisionmaking management of governments at all levels. At present, it has been commercialized in a number of central party and government departments and news media. Especially in the fields of land, public security, water conservancy, emergency, etc., it has become an indispensable support platform for business informatization. 
All the time, image service always stands at the heart of national node, which has kept the most calls and visits. With the release of multi-temporal image service, the new map has applied in several projects. As a consequence, it provides more image information and plentiful experience.

Land Resource Investigation has employed the multi-temporal image service, the users developed an app based on Android SDK, and the public and investigators use the app to submit some illegal land use (Figure13).
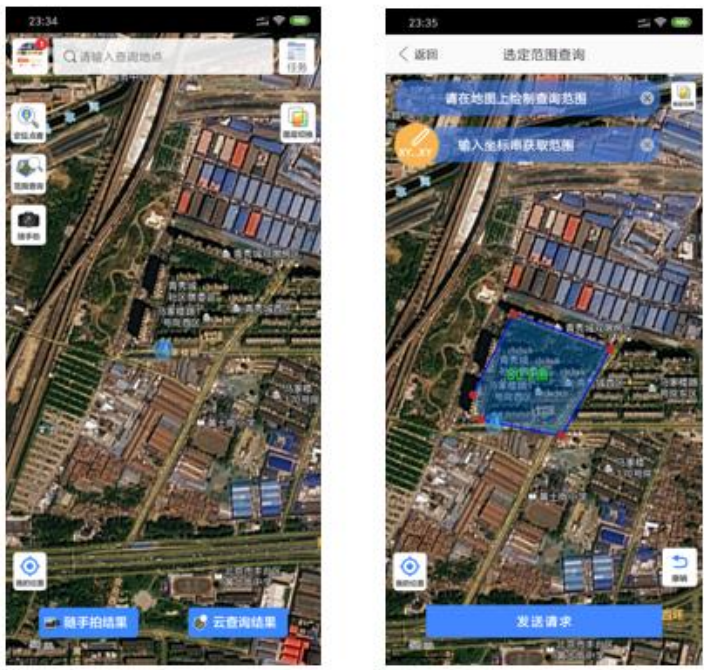

Figure13.Land Resource Investigation based on multitemporal image

The national pipeline mapping system (Figure 14) was developed based on this system. And it supported the geospatioal services for pipeline design, implementation and monitoring. As well as, the multi-temporal image could provide more details of the pipelines' locations, which could assist design and decision making.

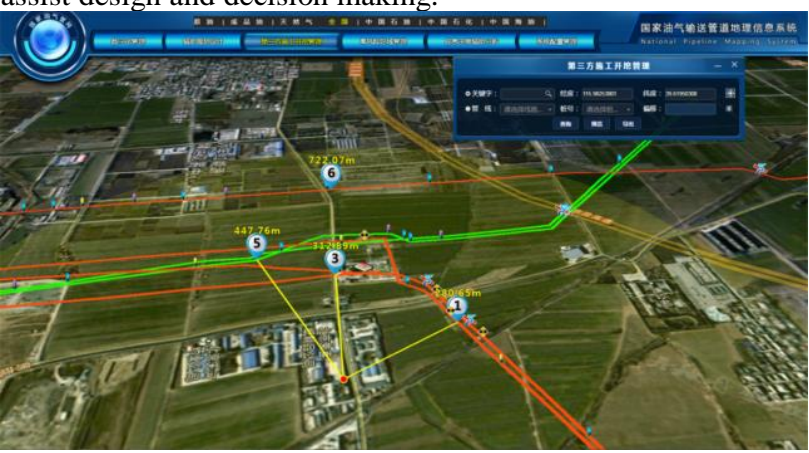

Figure14. National Pipeline Mapping System based on multitemporal image

Before the release of multi-temporal images, various types of users have put forward great demands for multi-temporal image data. Ordinary users hope to compare the changes of various features in the area they care about through images of different periods. The government hopes that the release of this service can provide more support to their decision-making.

The multi-temporal release meets the needs of the abovementioned different users. It also provides richer information and better experience for national platform's image service.

At present, some users have put forward more needs for multitemporal image services, including: reflecting the changes in urban agglomerations in the past decade or two, and the changes in famous elements such as the Yangtze River over the years. These feedbacks will also provide more ideas for the application of multi-temporal image services.

\section{RESULTS AND DISCUSSIONS}

The multi-temporal image service system has released image data from the year of 2013 to 2019, and the data size has reached 7TB. The system now can browse data in different time, and it provides query based on region, region code and time.

National Platform for Common Geospatial Information Services owns massive image data and ways to obtaining data. Based on using experience and feedback of users, there are a few of discussions about this research.

Firstly, this service focuses on the tiles data, however, it isolated to the sensing image data of accumulation. From view of image data production and fusion, the management of whole production process and remote sensing image data could be contributed to national platform, which has massive data and various data resources.

Secondly, the advantage of employing tiles data is reduce the size of data. Nevertheless, there is still one disadvantage for this method. The edge places of different update batch are divided by tiles, which could not corresponded with the real metadata information, such as image acquisition time. That will be researched deeply in the future.

Thirdly, the first vision of multi-temporal image map released only part of image data of national platform. However, as the national node, image data of level 10 to level 16 (scale from 1:577791 to 1:9027) will be released in the next version. These data includes various resources, such as Resources satellite three (ZY-3), Gaofen-1 satellite (GF-1), Gaofen-102C satellite and etc., which are mostly researched and developed domestically. Furthermore, this plan will bring massive historical image back into active use in the future work.

Next, prospective standards for data should be set. With the development of national platform, technical standards kept processing almost every year. But there has been less consideration on the historical data. So technical standards should be compatible different versions of historical standards.

The last point is to obtain data widely. Rich and diverse data is an important aspect for enriching online services. After years of development, although national platform has multiple ways to obtain data, $90 \%$ of the image data is obtained through data fusion projects. The data resources obtained through this way are relatively simple, and the production cycle is also long, which led to the dilemma of releasing the data of the previous year that year. Therefore, broadening data acquisition channels and improving publishing efficiency will also become the focus of the next step.

The important role of National Platform for Geospatial Information Services in China is self-evident. As the builder of national platform, the above discussions and thinking will combine application requirements and technological development for further practice. Multi-temporal image service, as one of the important services of the national form, will also use richer data information, more powerful and more stable services to help the development of national form. At the same time, the builders of the national platform must have a higher standing position. On the one hand, it continues to explore the applications that show China's development and construction. On the other hand, it can provide corresponding services for the people's daily needs. 


\section{ACKNOWLEDGEMENTS}

The work was supported by National Key Research and Development Program of China (No. 2017YFB0503705).

\section{REFERENCES}

Chen, A., 2019: Design and Implementation of Massive Remote Sensing Image Management System. Geomatics \&Spatial Information Technology, 42(10), 145-146.

Hou, P., Chen, L., Cheng, G., 2010: A New Method of MultiTemporal Remote Sensing Images Storage Management. Ordnance Industry Automation, 29(3), 63-67.

Jiang, J., Wu, H., Huang, W., 2017: Key Techniques and Project Practice for Establishing National Geo-Information Service Platform " Tianditu”. Acta Geodaetica et Cartographica Sinaica, 46(10), 1665-1667.

Liu, Q., Zhou, Q., Su, Y., Li, G., 2016: The Development and Strategic Value of National Geographic Information Public Service Platform. Geomatics \& Spatial Information Technology, 39(7):101-103.

Wang, L., Tang, J., Miao, J., 2014: Research on Management and Sharing of Large-Scale Image Based on ArcGIS. Advances in Geosciences, 4(1), 16-21.

The PostgreSQL Global Development Group, 1996-2021. Version 10.0. https://www.postgresql.org/.

Wang, M., Pan, J., 2004: Technique of Image Block Coding For Multi-temporal Image Management. Journal of Geomatics, 1007-3817(2004)03-0024-02.

Wei, X., Wu, K., Zhu, C., Liu, T., 2015: Design and Implementation of Remote Sensing Image Data Management and Analysis System. Urban Geotechnical Investigation \& Surveying, 29(3), 24-25.

Wikipedia contributors. 2018. Elasticsearch. (2018). Retrieved May 06, 2018 from https://en.wikipedia.org/wiki/Elasticsearch.

Yang, R., Bai, J., Huang, Z., Wu, M., Fan, R., 2011: Design and Implementation of a Massive Image Data Management System Based on SQLite. Computer Engineering \& Science, 33(10), 140-144. 